\title{
An Evidence of Esophageal Decompensation in Patients With Achalasia in the View of Its Subtype: A Retrospective Study
}

\author{
Kwangwon Rhee, Hanho Jeon, Jie-Hyun Kim, Young Hoon Yoon, Hyojin Park* and Sang In Lee
}

Department of Internal Medicine, Gangnam Severance Hospital, Yonsei University College of Medicine, Seoul, Korea

\begin{abstract}
Background/Aims
Achalasia is a primary motility disorder of esophagus. Many parameters represent esophageal function and morphologic changes, but their interrelationship is not yet established. We hypothesized that esophageal body would need to generate unusual pressure to empty the food bolus through the non-relaxing lower esophageal sphincter in patients with achalasia; therefore, higher is the residual lower esophageal sphincter pressure, greater would be the contraction pressure in the esophageal body in these patients. To verify the hypothesis, correlations among parameters from esophageal manometry, esophagography and esophageal transit study had been investigated.
\end{abstract}

\section{Methods}

A retrospective review of 34 patients was conducted. Resting lower esophageal sphincter pressure and contraction pressure of esophageal body were obtained from conventional esophageal manometry. Diameter of esophageal body was measured from barium column under esophagography. Radionuclide imaging was performed to assess the esophageal transit, designated as $R_{30}$, which was the residual radioactivity at 30 seconds after ingesting radioactive isotope.

Results

In vigorous achalasia group, contraction pressure of esophageal body was negatively correlated to dilated diameter of esophageal body $(P=0.025$, correlation coefficient $=-0.596)$. Esophageal transit was more delayed as dimensions of esophageal body increased in classic achalasia group $(P=0.039$, correlation coefficient $=0.627)$.

\section{Conclusions}

Diameter of esophageal body in classic achalasia was relatively wider than that of vigorous achalasia group and the degree of delayed esophageal transit was proportionate to the luminal widening. Patients with vigorous achalasia had narrower esophageal lumen and relatively shorter transit time than that of classic achalasia group. Proper peristalsis is not present in achalasia patients but remaining neuromuscular activity in vigorous achalasia patients might have caused the luminal narrowing and shorter transit time.

(J Neurogastroenterol Motil 2013;19:319-323)

Key Words

Esophageal achalasia; Manometry; Radionuclide imaging

Received: October 13, 2012 Revised: April 22, 2013 Accepted: April 30, 2013

(c) This is an Open Access article distributed under the terms of the Creative Commons Attribution Non-Commercial License (http://creativecommons. org/licenses/by-nc/3.0) which permits unrestricted non-commercial use, distribution, and reproduction in any medium, provided the original work is properly cited.

*Correspondence: Hyojin Park, MD, PhD

Professor of Medicine, Department of Internal Medicine, Gangnam Severance Hospital, Yonsei University, 211, Eonju-ro, Gangnam-gu, Seoul, Korea

Tel: +82-2-2019-3318, Fax: +82-2-3463-3882, E-mail: hjpark21@yuhs.ac.kr

Financial support: None.

Conflicts of interest: None.

Author contributions: Kwangwon Rhee wrote the manuscript, collected and interpreted the data. Hanho Jeon, Jie-Hyun Kim, Young Hoon Yoon and Sang In Lee collected and analyzed the data. Hyojin Park planned and conducted the study and reviewed the data and manuscript. 


\section{Introduction}

Study of esophageal motility entered a new era with the advent of high-resolution manometry. The new modality is unmatchable to conventional method in its accuracy and precision. High-resolution manometry enabled re-definition or re-classification of motility diseases of esophagus which had originally been identified with conventional manometry. Researchers gathered information and understood the nature of the motility diseases of esophagus by conventional manometry in the past. With large amount of data accumulated since its introduction, review of data from conventional manometry may still hold some value. Among the diseases undergoing changes, achalasia is one of them. Achalasia is a progressive disease without proper peristalsis and with abnormal relaxation of the lower esophageal sphincter (LES). Some researchers considered contraction pressure of 37 $\mathrm{mmHg}$ by esophageal body to be the main criteria in categorizing the disease into classic and vigorous types although the exact pressure at which to divide them into 2 groups did not reach consensus. $^{1-3}$ On the spectrum of decompensation, vigorous achalasia represents the acute form with remnant neuromuscular activity in the esophageal body, while classic achalasia is taken as the chronic form with dilated esophageal body, followed by muscular weakness. ${ }^{4}$ Improper relaxation of LES in achalasia patients worsens food stasis. ${ }^{5}$ We hypothesized that this stasis may cause compensatory increase in contraction pressure of esophageal body to pass stagnated food material through the esophagogastric junction. Retrospective review of conventional manometric data was conducted. Previous studies demonstrated that esophageal luminal widening worsens with the progression of disease, ${ }^{6}$ but interrelationships of esophageal lumen with other parameters have not been studied. Widths of the esophageal lumen, the resting LES pressure, and esophageal transit parameter have been analyzed in classic and vigorous achalasia to verify the hypothesis.

\section{Materials and Methods}

A retrospective review of 140 consecutive patients who visited Gangnam Severance Hospital, from January 2004 to October 2011 was conducted. Previously treated 27 patients with classic achalasia, 17 with vigorous achalasia and 64 patients without esophageal manometry data were excluded. 34 untreated achalasia patients were reviewed. Patients, with abnormal relaxation of LES and without proper peristalsis under conventional esophageal manometry, were diagnosed as achalasia. Patients were also evaluated by esophagography and radionuclide imaging. ${ }^{7}$

\section{Esophageal Manometry}

Esophageal manometry was performed with a low-compliance, pneumohydraulic capillary infusion system (Arndorfer Medical Specialities, Greendale, WI) using 8-channel water-perfused catheters. Catheters were gently inserted manually after lubrication. After measuring resting LES pressure, contraction pressure of esophageal body was measured 5 centimeters above the LES during 10 wet swallows at 30-second intervals. Pressures were digitized and recorded by a PC polygraf HR system (Medtronic Synectics Medical, Minneapolis, Minnesota, USA). Pressures of $37 \mathrm{mmHg}$ or higher were categorized as vigorous achalasia, and lower pressures as classic achalasia. ${ }^{1-3}$

\section{Esophagography}

Before esophagography, patients were instructed to hold oral intake for more than 9 hours. Esophagography was performed in the erect anteroposterior projection, as well as in the erect left posterior and anterior oblique projections under fluoroscopy (Shimavision 2000HG; Shimadzu, Kyoto, Japan). 120 mL barium sulphate was prepared at concentration of $140 \% \mathrm{w} / \mathrm{v}$. Esophageal lumen was observed fluoroscopically during 3 to 4 mouthful swallows in 5 seconds interval, and a series of spot images were obtained 1,2 and 5 minutes after complete swallow. The barium esophagographic studies were reviewed by a gastrointestinal radiologist. Due to the retrospective nature of this study, different levels of magnification and various photographic viewing angles necessitated standardized quantification. Hence, an esophageal width ratio was adopted to evaluate the dimensions of the esophageal body by dividing the maximum diameter of the planes perpendicular to esophageal axis of the barium column by the minimum width of the resting esophagogastric junction ${ }^{8}$ (Figure). Measurement of the maximal caliber of esophageal body and the width of resting esophagogastric junction was performed on picture archiving and communicating system (PACS) images.

\section{Esophageal Transit Scintigraphy}

Esophageal transit scintigraphy was performed with technetium-99m after overnight fasting. A gamma camera (Genesys Vertex; AdacLab., Milpitas, CA, USA) with a large field of view was used, and anterior scans were obtained in the erect position. 


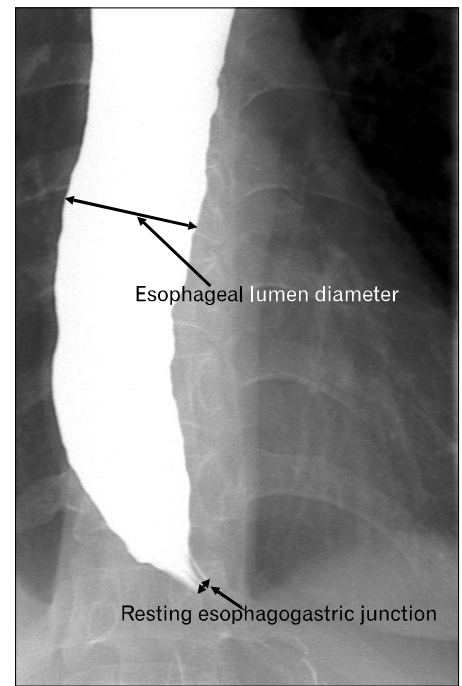

Figure. Esophageal width ratio is calculated by dividing the esophageal lumen diameter by the resting esophagogastric junction.

At 0 second, $111 \mathrm{MBq}(3 \mathrm{mCi})$ of technetium-99m pertechnetate suspended in $35 \mathrm{~mL}$ of water was orally administered on command while the patient was in the upright position, and the patient was told not to swallow again until directed. Dynamic images of the esophageal area were acquired for 5 minutes at a rate of 120 shots per minute, and static images were taken at 30 seconds after radioisotope ingestion. Maximal radioactivity and the radioactivity 30 seconds after the peak were measured. $R_{30}$ was defined as the percentage of residual radiation in the distal esophagus 30 seconds after the peak radioactivity.

\section{Statistical Methods}

To compare the baseline characteristics of classic and vigorous achalasia, Mann-Whitney U tests were used. Bivariate correlations were used to determine associations between the parameters: resting LES pressure, mean contraction pressure of esophageal body from esophageal manometry, esophageal width ratio from esophagography, and $\mathrm{R}_{30}$ from esophageal transit scintigraphy. Spearman's rho was used due to non-normally distributed data. All values were expressed as median (range), and results were considered statistically significant if $P$-values were lower than 0.05 .

\section{Results}

Demographic characteristics, and median values and range of the parameters of esophageal motor function are shown in Table 1. Of the 34 patients with achalasia, 17 (50\%) had classic
Table 1. Demographics and Baseline Characteristics of Classic and Vigorous Achalasia

\begin{tabular}{lccc}
\hline Characteristics & $\begin{array}{c}\text { Classic achalasia } \\
(\mathrm{n}=17)\end{array}$ & $\begin{array}{c}\text { Vigorous achalasia } \\
(\mathrm{n}=17)\end{array}$ & $P$-value \\
\hline Sex (M/F) & $5 / 12$ & $6 / 11$ & 0.714 \\
Age (years) & 50 & 45 & 0.469 \\
& $(23-74)$ & $(26-64)$ & \\
Resting LES & 47.0 & 52.2 & 0.399 \\
pressure & $(22.6-130.0)$ & $(22-121.6)$ & \\
Contraction & 21.6 & 56.9 & $<0.001$ \\
pressure of & $(15.1-36.7)$ & $(40.4-154.0)$ & \\
esophageal body & & & \\
Esophageal & 20.9 & 8.6 & 0.002 \\
width ratio & $(4.4-39.7)$ & $(4.7-21.0)$ & \\
$\mathrm{R}_{30}(\%)$ & 81.5 & 60.9 & 0.001 \\
& $(37.0-99.0)$ & $(10.0-76.0)$ & \\
\hline
\end{tabular}

LES, lower esophageal sphincter.

Data are presented as median (range). $P$-value $<0.05$ is considered significant.

Table 2. Correlations of Resting Lower Esophageal Sphincter Pressure With Body Pressure and Esophageal Width Ratio, Comparing Classic and Vigorous Achalasia

\begin{tabular}{lcc}
\hline vs. Resting LES pressure & Classic achalasia & Vigorous achalasia \\
\hline $\begin{array}{c}\text { Contraction pressure of } \\
\text { esophageal body }\end{array}$ & $0.277(0.282)$ & $0.044(0.866)$ \\
Esophageal width ratio & $-0.024(0.935)$ & $-0.235(0.418)$ \\
\hline
\end{tabular}

LES, lower esophageal sphincter.

Data are presented as correlation coefficient $(P$-value). $P$-value $<0.05$ is considered significant.

type and the other 17 had vigorous type. The median age for classic achalasia was 50 (range 23-74 years), and for vigorous achalasia, it was 45 (range 26-64 years). Median ages and gender compositions of the 2 groups did not differ significantly. There was no significant difference in the resting LES pressures of patients with classic versus vigorous achalasia, which were 47.0 $\mathrm{mmHg}$ (22.6-130.0 $\mathrm{mmHg}$ ) and $52.2 \mathrm{mmHg}(22.0-121.6$ $\mathrm{mmHg})$, respectively $(P=0.399)$. However, the esophageal width ratio in classic achalasia was 20.9 (4.4-39.7) which was higher than in vigorous achalasia $8.6(4.7-21.0)(P=0.002)$. Additionally, during esophageal scintigraphy, the $\mathrm{R}_{30}$ was significantly higher in classic achalasia than in vigorous achalasia, which were $81.5 \%(37.0-99.0 \%)$ and $60.9 \%$ (10.0-76.0\%), respectively $(P=0.001)$.

Bivariate correlational analysis found little significant associations among the studied parameters in our sample. Specifically, the relationship between resting LES pressure and contraction 
Table 3. Correlations of $\mathrm{R}_{30}$ With Body Pressure, Resting Lower Esophageal Sphincter Pressure and Esophageal Width Ratio, Comparing Classic and Vigorous Achalasia

\begin{tabular}{ccc}
\hline vs. $\mathrm{R}_{30}$ & Classic achalasia & Vigorous achalasia \\
\hline $\begin{array}{c}\text { Contraction pressure of } \\
\text { esophageal body }\end{array}$ & $0.209(0.474)$ & $0.150(0.579)$ \\
Resting LES pressure & $0.288(0.305)$ & $-0.256(0.339)$ \\
Esophageal width ratio & $0.627(0.039)$ & $0.434(0.138)$ \\
\hline
\end{tabular}

LES, lower esophageal sphincter.

Data are presented as correlation coefficient ( $P$-value). $P$-value $<0.05$ is considered significant.

pressure of esophageal body was not significant in either classic ( $P=0.282)$ or vigorous achalasia $(P=0.866)$. The esophageal width ratio was also not significantly correlated with resting LES pressure in both groups $(P=0.935$ in classic achalasia group and $P=0.418$ in vigorous achalasia group) (Table 2). Pressure parameters and esophageal dimensions did not have a significant influence on esophageal transit. Contraction pressure of esophageal body $(P=0.474)$ and resting LES pressure $(P=0.305)$ were not significantly related to $R_{30}$ in classic achalasia. $R_{30}$ in vigorous achalasia did not demonstrate any proportionate increase or decrease with contraction pressure of esophageal body $(P=0.579)$ and resting LES pressure $(P=0.339)$. However, a positive correlation existed between esophageal width ratio and $\mathrm{R}_{30}$ in classic achalasia $(r=0.627, P=0.039)$, which was not seen in vigorous achalasia $(P=0.138)$ (Table 3$)$. In addition, ratio of esophageal dimensions was negatively correlated with contraction pressure of esophageal body in vigorous achalasia $(r=-0.596, P=0.025)$, while classic achalasia did not show any significant relationship $(P=0.817)$ (Table 4).

\section{Discussion}

Chicago classification divides achalasia into 3 groups with high-resolution manometry. In type I, there is no pressurization and it is compatible with classic achalasia and achalasia with compression is categorized as type II. Type III is achalasia with spasm which resembles vigorous achalasia. ${ }^{9}$ On the other hand, conventional manometry categorized achalasia into 2 groups which are classic and vigorous achalasia by contraction pressure of esophageal body. ${ }^{2}$

Relationships among parameters of esophagus, which include resting LES pressure, contraction pressure of esophageal body, esophageal width ratio and $\mathrm{R}_{30}$, were investigated. The
Table 4. Correlation of Contraction Pressure of Esophageal Body With Esophageal Width Ratio

\begin{tabular}{lcc}
\hline vs. Esophageal width ratio & Classic achalasia & Vigorous achalasia \\
\hline $\begin{array}{c}\text { Contraction pressure of } \\
\text { esophageal body }\end{array}$ & $-0.068(0.817)$ & $-0.596(0.025)$ \\
\hline
\end{tabular}

Data are presented as correlation coefficient $(P$-value $) . P$-value $<0.05$ is considered significant.

analysis demonstrated that the esophageal width ratio was bigger and the transit was slower in classic achalasia. Decompensation of esophageal body in classic achalasia might have caused slower transit and luminal dilatation. Three patients had mega-esophagus in classic achalasia group while none was observed in vigorous group. These are compatible with common notion that classic achalasia signifies chronic form. On the other hand, relatively narrower esophageal body and shorter transit time of vigorous achalasia patients may be result of residual contractility.

A hypothesis was formulated that abnormal relaxation of LES would provoke the esophageal body to generate more force in an effort to pass intraluminal material through the LES. However, no significant correlation between contraction pressure of esophageal body and resting LES pressure was observed (Table 2). This may be attributable to the various degrees of esophageal decompensation among individuals. In classic achalasia, the esophageal body has lost most of its neuromuscular activity, and it remains unresponsive to a blockade of the esophagogastric junction. Esophageal body of vigorous achalasia has remaining contractile force, which may be expected to demonstrate proportional rise with resting LES pressure. But statistical significance was not present. Since vigorous achalasia is also on a spectrum of transforming into classic achalasia, certain degree of decompensation exist in vigorous achalasia. ${ }^{10}$ Therefore, in our sample, this diverse degree of decompensation could have precluded proportionate relationship. Esophageal width ratio may be in a similar situation. If esophageal body of all the classic achalasia patients had completely lost all neuromuscular activity, then an increase in resting LES pressure would directly lead to proportional luminal widening. However, since patients with classic achalasia have various degrees of remnant neuromuscular function, this may have made correlation between contractions of the esophageal body and resting LES pressure impossible. Although those contractions might be minor in classic achalasia, they should have been sufficient to bring statistical non-significance.

Resting LES pressure and esophageal transit demonstrated 
no correlation. Such result is consistent with the finding that resting LES pressure is not significantly correlated with contraction pressure of esophageal body. Contraction pressure of esophageal body had no statistically significant influence on esophageal transit in both groups as well, as depicted in Table 3. However, vigorous achalasia group had significantly shorter transit time than classic achalasia, as indicated by $\mathrm{R}_{30}$ in Table 1 . Tertiary contractions in vigorous achalasia may have facilitated the passage of radioactive isotope through the GE junction even in the absence of proper peristalsis. Such a relationship is supported by the association of contraction pressure of esophageal body with ratio (Table 4). Less decompensated esophageal body of vigorous achalasia had contractility and passed barium into gastric lumen, which lead to smaller esophageal dimensions. On the other hand, esophageal width ratio was positively correlated with $\mathrm{R}_{30}$ in classic achalasia, indicating that wider dimension is directly related to slower transit. Increasing degree of decompensation in classic achalasia might have caused the expansion of esophageal lumen along with stagnation of barium.

Constant debate went on regarding the necessity of distinction between classic and vigorous achalasia in the era of conventional manometry. ${ }^{4}$ The term, vigorous achalasia, which was defined by the high contraction pressure of esophageal body, encompassed both achalasia with compression and spasm. ${ }^{11}$ Type II achalasia with low compression pressure could have been defined as the classic achalasia. Such dubious classification might have caused lack of correlation between reviewed parameters. Introduction of high-resolution manometry have made differentiation among type I, type II and type III feasible. A prospective study with new classification could be conducted to reveal relationships among functional, radiological and manometrical parameters.

This study set out to establish some relationships between esophageal parameters thereby provide some clinically significant information, but the results do not show much correlation. Lack of inter-relationship among pressure, transit, and dimension may have resulted from various degrees of decompensation within each individual. If so, symptom duration might provide additional information on the degree of neuron degeneration, which in turn could represent the degree of decompensation especially in type II. Retrospective nature limited acquirement of precise data regarding patient history and symptoms. Future study with high resolution manometry should be conducted in a prospective design with thorough history taking to better evaluate the extent of decompensation.

\section{References}

1. Jung KW, Jung HY, Yoon IJ, et al. Basal and residual lower esophageal pressures increase in old age in classic achalasia, but not vigorous achalasia. J Gastroenterol Hepatol 2010;25:1452-1455.

2. Goldenberg SP, Burrell M, Fette GG, Vos C, Traube M. Classic and vigorous achalasia: a comparison of manometric, radiographic, and clinical findings. Gastroenterology 1991;101:743-748.

3. Camacho-Lobato L, Katz PO, Eveland J, Vela M, Castell DO. Vigorous achalasia: original description requires minor change. $\mathrm{J}$ Clin Gastroenterol 2001;33:375-377.

4. Richter JE. Achalasia - an update. J Neurogastroenterol Motil 2010;16:232-242.

5. Holloway RH, Krosin G, Lange RC, Baue AE, McCallum RW. Radionuclide esophageal emptying of a solid meal to quantitate results of therapy in achalasia. Gastroenterology 1983;84:771-776.

6. Shiino Y, Houghton SG, Filipi CJ, Awad ZT, Tomonaga T, Marsh RE. Manometric and radiographic verification of esophageal body decompensation for patients with achalasia. J Am Coll Surg 1999; 189:158-163.

7. Chung JJ, Park HJ, Yu JS, et al. A comparison of esophagography and esophageal transit scintigraphy in the evaluation of usefulness of endoscopic pneumatic dilatation in achalasia. Acta Radiol 2008;49: 498-505.

8. Moon JT, Jung IS, Kim YS, Cho SH, Park H, Lee SI. Correlation between clinical symptoms and radiologic findings before and after pneumatic balloon dilatation for achalasia. Korean J Gastroenterol 2008;52:16-20.

9. Bredenoord AJ, Fox M, Kahrilas PJ, et al. Chicago classification criteria of esophageal motility disorders defined in high resolution esophageal pressure topography. Neurogastroenterol Motil 2012; 24(suppl 1):57-65.

10. Millan MS, Bourdages R, Beck IT, DaCosta LR. Transition from diffuse esophageal spasm to achalasia. J Clin Gastroenterol 1979;1: 107-117.

11. Pandolfino JE, Kwiatek MA, Nealis T, Bulsiewicz W, Post J, Kahrilas PJ. Achalasia: a new clinically relevant classification by highresolution manometry. Gastroenterology 2008;135:1526-1533. 